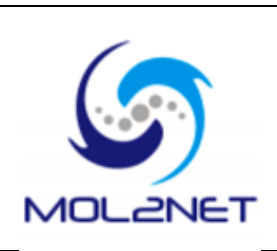

\title{
Dual-modality probe for MRI and photoacoustic imaging: synthesis, and relaxometric characterization
}

\author{
Marie Devreux ${ }^{1}$, Fabienne Dioury ${ }^{2, *}$, Céline Henoumont ${ }^{1}$, Clotilde Ferroud ${ }^{2}$ and \\ Sophie Laurent ${ }^{1,3, *}$ \\ 1 General, Organic and Biomedical Chemistry Unit, NMR and Molecular Imaging Laboratory, 19 \\ Avenue Maistriau B-7000 Mons, Belgium ; E-Mails: marie.devreux @ student.umons.ac.be (MD) \\ celine.henoumont@umons.ac.be (CH); sophie.laurent@umons.ac.be (SL)
}

2 Hesam Université, Conservatoire national des arts et métiers, Laboratoire CMGPCE, 2 rue Conté, F-75003 Paris, France ; E-Mail: fabienne.dioury@lecnam.net (FD) ; clotilde.ferroud@lecnam.net (CF)

3 Center for Microscopy and Molecular Imaging, 8 rue Adrienne Bolland B-6041 Charleroi, Belgium

* Authors to whom correspondence should be addressed; E-Mail: fabienne.dioury@lecnam.net ; sophie.laurent@umons.ac.be

Tel.: +33140272948; +3265 373525

Received: / Accepted: / Published:

\begin{abstract}
Multimodality imaging based on complementary modalities is a way to improve the accuracy of medical diagnoses. For such purpose, multimodal probes combining the appropriate contrastophores into a single delivery are required. The project presented here deals with the combination of two modalities: magnetic resonance imaging (MRI), and photoacoustic imaging (PAI). The dual probe envisaged relies on a pyclen-based gadolinium(III) complex as contrast agent for MRI, together with ZW800-1 fluorophore for PAI, both of them being grafted on a linker derived from $L$ lysine. The results presented here describe the synthetic pathway, and the relaxometric characterization of the dual compound.
\end{abstract}

Keywords: medical imaging; molecular imaging; multimodality; MRI; photoacoustic; gadolinium; bifunctional chelating agents; pyclen; PCTA12; ZW800-1.

\section{Introduction}

Magnetic resonance imaging (MRI) is a noninvasive diagnostic modality of high spatial resolution $(10 \mu \mathrm{m}-1 \mathrm{~mm})$ widely used in clinical medicine to visualize soft tissues and obtain anatomical information. For better accuracy, a paramagnetic contrast agent (CA), most often a complex of gadolinium, can be administrated to overcome the intrinsic low sensitivity of MRI $(\sim 100 \mu \mathrm{M})$ and improve the contrast of the images. This enhancement is however limited by the possibly injectable dose of CA $(0,1 \mathrm{mmol} / \mathrm{kg})$ so that alternative or complementary solutions may be developed. Multimodality imaging that associates complementary techniques is a way to overcome some intrinsic weaknesses of a given technique; for such purpose, multimodality probes that combine several contrastophores in a single delivery are required. 
Photoacoustic imaging (PAI) is another noninvasive modality whose penetration depth is compatible with clinical applications (up to 4 $\mathrm{cm})$. Moreover, with lower spatial resolution (75 $\mu \mathrm{m})$ but higher sensitivity $(\sim 100 \mathrm{pM})$ than MRI, combining these two modalities appears valuable to compensate the respective weakness of each one. In that project, we propose a dual-modality probe built by connection of two contrastophores: a pyclen-based gadolinium complex derived from PCTA12 [1] (Figure 1) as CA for MRI, and the near-infrared (NIR) fluorophore ZW800-1 [2] based on the indocyanine green (ICG) structure. Moreover, $L$ lysine was chosen as trifunctionalizable linker to graft the two imaging counterparts, and to allow as well subsequent conjugation to a targeting ligand (biomolecule) as vehicle with specific tropism for a targeted pathological tissue (molecular imaging).
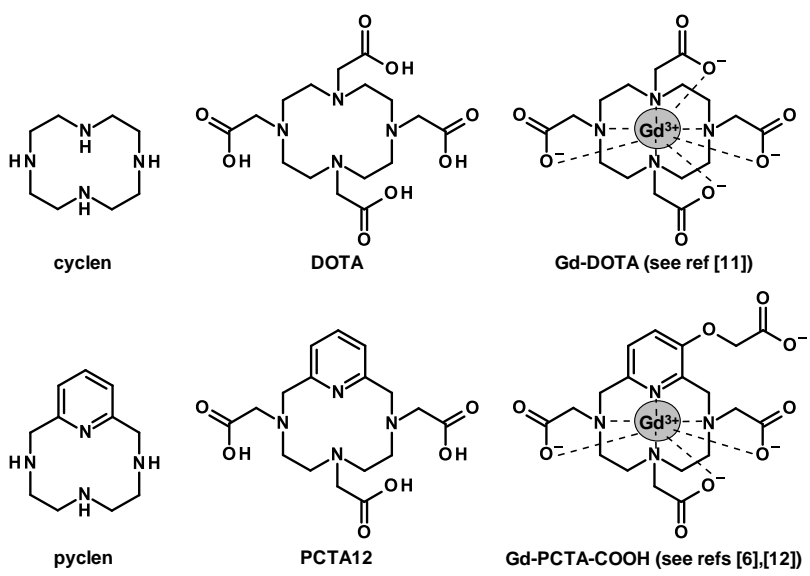

Figure 1. 12-membered azamacrocycles, acetate $N$-functionalized polyazamacrocyclic ligands for $\mathrm{Gd}(\mathrm{III})$, and Gd-complexes used as references for the relaxometric studies.

\section{Results and Discussion}

\section{Dual-modality probe synthesis}

PCTA12 is a rigid analogue of DOTA (Figure 1) known to form very stable complex with $\mathrm{Gd}^{3+}(\log k \sim 20-21)$, with high kinetic inertness,[3][4] and water relaxivity higher than that of other clinically approved CAs for MRI ( $r_{1}$ $=5.4 \mathrm{~s}^{-1} \mathrm{mM}^{-1}$ at $310 \mathrm{~K}$ and $20 \mathrm{MHz}$ ), [5] thus displaying promising potentialities in the field of molecular imaging.[6]

The zwitterionic NIR fluorophore ZW800-1 is a ICG derivative exhibiting low background and outperforming the signal-to-background ratio of some other commercially available NIR fluorophores thus being promising as well for diagnostic applications.[2][7]

Our synthesis began by the preparation of the linker. Selective functionalization of $L$-lysine is required to allow sequential grafting of the different parts of the targeted probe (Scheme 1). The selective carboxybenzyl $(\mathrm{Cbz})$ protection of the primary amine of the lateral chain was readily achieved according to a literature protocol.[8a] The protection of the carboxylic acid was then done in allyl alcohol medium by slight modification of some reported thionyl chloride methods.[8] The subsequent conjugation to the functionalizable precursor of the chelating agent, namely PCTA-tris(tBu)-COOH previously prepared in our lab [6], proceeded on the free $\alpha$ amino site under "one-pot" uronium activation using HBTU as coupling agent. The cleavage of both tert-butyl esters and Cbz amine's protection occurred in acidic medium, and was followed by the complexation of $\mathrm{Gd}^{3+}$ performed as previously described for PCTA12-type chelating agent by mixing an equimolar amount of gadolinium chloride in warmed $\mathrm{pH}$-controlled aqueous solution.[9]
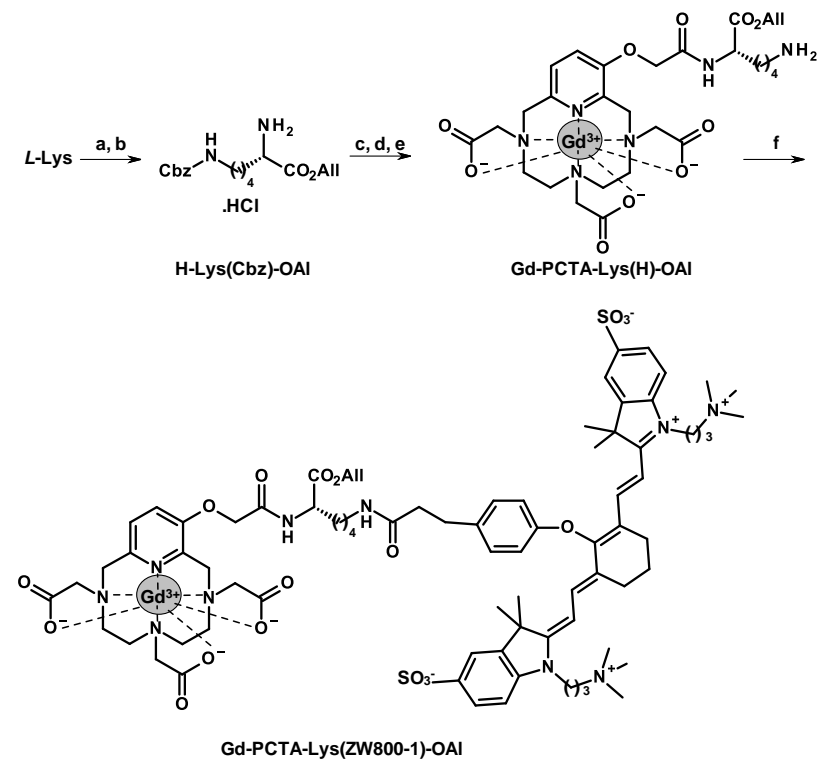

Scheme 1. Dual-modality probe synthesis. Reagents and conditions: (a) See ref [8]; (b) $\mathrm{SOCl}_{2}$, allyl alcohol (CAS RN 107-18-6); (c) PCTA-tris(tBu)-COOH (see ref [6]), HBTU (CAS RN 94790-37-1), DIPEA, $\mathrm{CH}_{2} \mathrm{Cl}_{2}$; (d) TFA (CAS RN 76-05-1), $\mathrm{CH}_{2} \mathrm{Cl}_{2}$; (e) $\mathrm{GdCl}_{3}$, water, $\mathrm{pH} \sim 5.5$; (f) ZW800-1 (see ref [2]), 
EDC.HCl (CAS RN 25952-53-8), water, pH 5.5 .

ZW800-1 coupling then proceeded smoothly under conventional amide bond formation methodology involving previous activation with the water soluble carbodiimide EDC in a $p H$ controlled aqueous medium. The reaction was monitored by mass spectroscopy; ESI-TOF MS analysis was consistent with the expected probe Gd-PCTA-Lys(ZW800-1)-OAl giving two characteristic isotopic patterns corresponding to $[\mathrm{M}+\mathrm{H}]^{2+}$ and $[\mathrm{M}+\mathrm{Na}]^{2+}$ species (Figure 2).

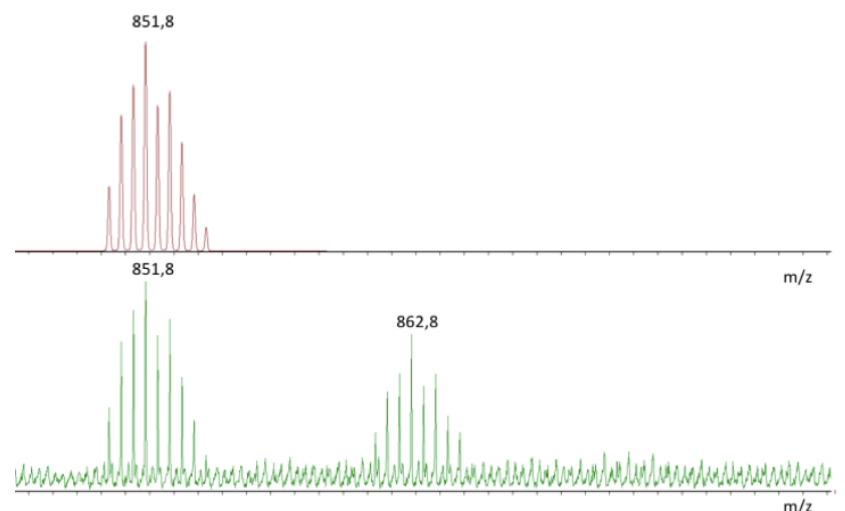

Figure 2. ESI-TOF MS spectra. Up: simulated on the basis of the empirical formula $\mathrm{C}_{79} \mathrm{H}_{103} \mathrm{~N}_{10} \mathrm{O}_{18} \mathrm{~S}_{2} \mathrm{Gd}$; bottom: experimental (High definition MS performed on Synapt G2-Si Waters by J. De Winter, S $^{2}$ MOs Lab).

Relaxometric characterization of the $G d$ complexes

NMRD profiles were recorded for both of the new Gd-complexes prepared. By comparing Gd-PCTA-Lys(H)-OAl with Gd-PCTA-COOH (Figure 1), a damageable effect of the lysine linkage on the water relaxivity $r_{1}$ can be deplored.(Figure 3) On the contrary, an important increase of the relaxivity was observed after subsequent coupling of the fluorophore ZW800-1, especially at higher magnetic fields.

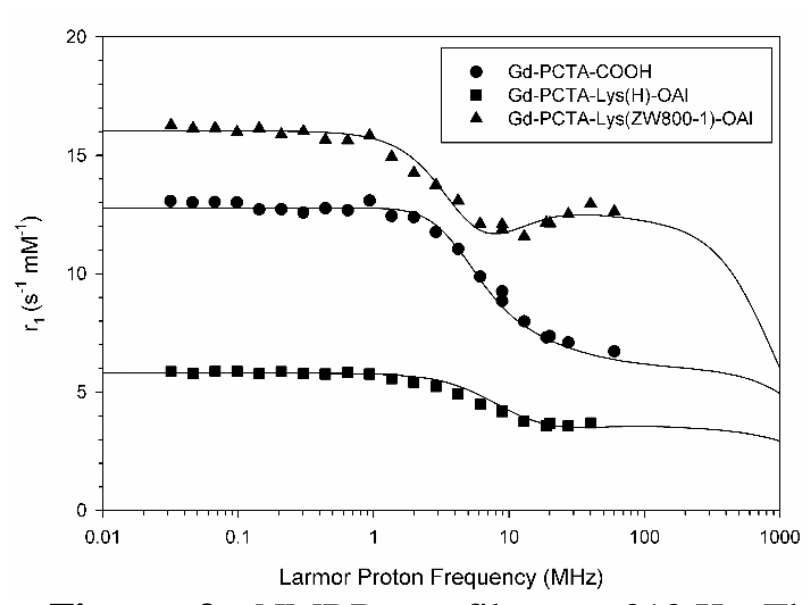

Figure 3. NMRD profiles at $310 \mathrm{~K}$. The straight lines correspond to the theoretical fitting according to the theory of Solomon and Bloembergen.[10]

In order to understand these results, the number of water molecules coordinated in the inner-sphere of gadolinium was measured by ${ }^{17} \mathrm{O}$ NMR. By comparison with known complexes Gd-PCTA-COOH [6a][12] and Gd-DOTA [11] having respectively two and one water molecules coordinated in their inner-sphere, it can be deduced that both of the new complexes grafted onto the lysine linker have only one water molecule in the coordination sites of the gadolinium ion (Figure 4). This can be rationalized by the additional coordination to the amide bond resulting from the coupling to the linker that increases the overall denticity of the chelating unit, and explaining the decrease of relaxivity for Gd-PCTA-Lys(H)-OAl compared to that for the parent Gd-PCTA-COOH. Consequently, it could anticipate that this quench of relaxivity could be avoided by lengthening the spacer between the macrocyclic chelating unit and the amide linkage (spacers longer than 3 atoms as for the O-acetyl unit of the present work). 


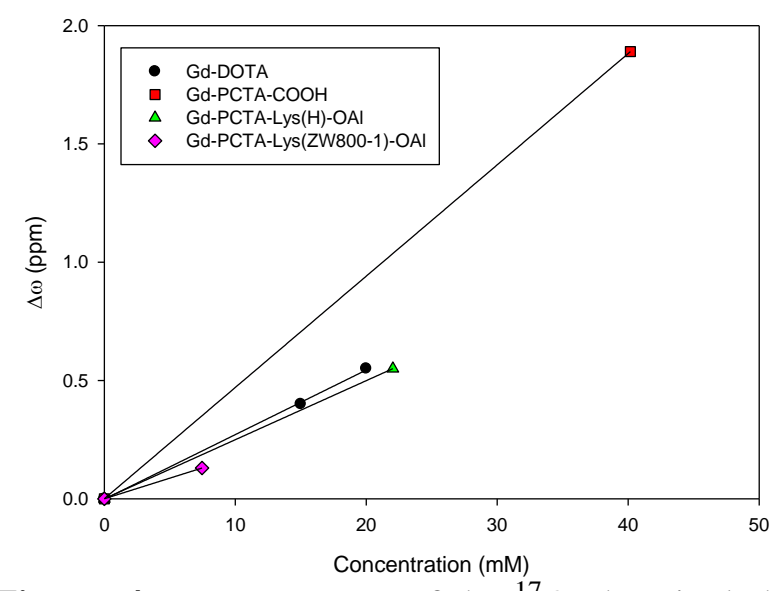

Figure 4. Measurement of the ${ }^{17} \mathrm{O}$ chemical shift of water in the presence of different Gdcomplexes.

The fitting of the NMRD curves with the well-known Solomon and Bloembergen theory brings more information about the observed

\section{Materials and Methods}

All the synthesized intermediates were characterized by ${ }^{1} \mathrm{H},{ }^{13} \mathrm{C}$ and $\mathrm{COSY}$ NMR (Bruker $^{\circledR}$ Avance II-500 at $500 \mathrm{MHz}$, Bruker ${ }^{\circledR}$ BioSpin $\mathrm{GmbH}$ at $400 \mathrm{MHz}$ or Bruker $^{\circledR} \mathrm{NEO}$ $600 \mathrm{MHz}$ ) and by mass spectrometry (Waters ZQ2000 or Synapt G2-Si Waters).

Proton nuclear magnetic relaxation dispersion (NMRD) profiles were measured on a Stelar Spinmaster FFC, fast field cycling NMR relaxometer (Stelar, Mede (PV), Italy) over a magnetic field strength range extending from $0.24 \mathrm{mT}$ to $0.7 \mathrm{~T}$. Measurements were performed on $0.5 \mathrm{~mL}$ samples contained in $7.5 \mathrm{~mm}$ o.d. relaxivities.[10] Among the different parameters influencing the relaxivity, a huge increase of the rotational correlation time $\tau_{R}$ was observed for the dual probe Gd-PCTA-Lys(ZW800-1)-OAl (179 ps) by comparison with that of the parent compounds (76, and $71 \mathrm{ps}$ for Gd-PCTA-COOH and Gd-PCTA-Lys(H)-OAl respectively). This can result from $\pi$-stacking intermolecular interactions that lead to a slow-moving system with favorable $\tau_{R}$ value, thus explaining the higher relaxivities of the dual probe by comparison with that for Gd-PCTA-COOH, especially between 20 and $60 \mathrm{MHz}$ where a slight hump can be observed on the NMRD profile.

pyrex tubes. Additional relaxation rates at 20, and $60 \mathrm{MHz}$ were respectively obtained on a Minispec mq20, and a Minispec mq60 (Bruker, Karlsruhe, Germany). The temperature was equal to $310 \mathrm{~K}$. Proton NMRD curves were fitted using data-processing software including different theoretical models describing the nuclear relaxation phenomena (Minuit, CERN Library). The ${ }^{17} \mathrm{O}$ chemical shifts were measured at $310 \mathrm{~K}$ and $\quad 11.75 \mathrm{~T}$ (Bruker AVANCEII-500 spectrometer) on solutions containing 8 to $40 \mathrm{mM}$ of the Gd-complex dissolved in $540 \mu \mathrm{l}$ of water added with $60 \mu$ of $\mathrm{D}_{2} \mathrm{O}$ for the lock.

\section{Conclusions}

These preliminary results validate the design of such dual-modality probe for MRI and PAI. However, they pointed out the influence of the nature of the spacer used to separate the chelating unit from the functionalizable linker: if too short, as for the 3-atoms spacer in the present work, the formed amide bond may act as an additional coordinating site thus increasing the overall denticity of the chelating unit, decreasing the number of water molecules in the inner-sphere of the paramagnetic species with damageable effect on its effectiveness as a CA for MRI.

\section{Acknowledgments}

This work was performed with the financial support of the FNRS, the ARC, the Walloon Region (Gadolymph, Holocancer and Interreg projects), the Interuniversity Attraction Poles of the Belgian Federal Science Policy Office and the COST actions. Authors thank the Center for Microscopy and Molecular Imaging (CMMI, supported by European Regional Development Fund and Wallonia). 
Thanks to PhD De Winter J. and Prof Gerbaux P. from $S^{2}$ MOs lab for the mass spectra.

\section{Author Contributions}

Conceived and designed the experiments: FD, CH, SL. Performed the experiments: MD, CH. Analyzed data: MD, FD, CH, SL. Contributed reagents/materials/analysis tools: CF, SL. Wrote the paper: MD, FD, CH, SL. All authors read and approved the final manuscript.

\section{Conflicts of Interest}

The authors declare no conflict of interest.

\section{References and Notes}

1. Stetter, H.; Frank, W.; Mertens, R. Darstellung und komplexbildung von polyazacycloalkan-Nessigsäuren. Tetrahedron 1981, 37, 767-772.

2. Choi, H. S.; Nasr, K.; Alyabyev, S.; Feith, D.; Lee, J. H.; Kim, S. H.; Ashitate, Y.; Hyun, H.; Patonay, G.; Strekowski, L.; Henary, M.; Frangioni, J. V. Synthesis and in vivo fate of zwitterionic near-infrared fluorophores. Angew Chem Int Ed Engl. 2011, 50(28), 6258-6263.

3. Aime, S.; Botta, M.; Geninatti Crich, S.; Giovenzana, G. B.; Jommi, G.; Pagliarin, R.; Sisti, M. Synthesis and NMR studies of three Pyridine-Containing Triaza Macrocyclic Triacetate ligands and their complexes with lanthanide ions. Inorg. Chem. 1997, 36, 2992-3000.

4. Tircso, G.; Benyo, E. T.; Suh, E. H.; Jurek, P.; Kiefer, G. A.; Sherry, A. D.; Kovacs, Z. (S)-5-( $p$ Nitrobenzyl)-PCTA, a promising bifunctional ligand with advantageous metal ion complexation kinetics. Bioconjugate Chem. 2009, 20, 565-575.

5. (a) Port, M.; Raynal, I.; Vander Elst, L.; Muller, R. N.; Dioury, F.; Ferroud, C.; Guy, A. Impact of rigidification on relaxometric properties of a tricyclic tetraazatriacetic gadolinium chelate. Contrast Media Mol. Imaging 2006, 1(3), 121-127; (b) Aime, S.; Botta, M.; Crich, S. G.; Giovenzana, G.; Pagliarin, R.; Sisti, M.; Terreno, E. NMR Relaxometric studies of Gd(III) complexes with heptadentate macrocyclic ligands. Magn. Reson. Chem. 1998, 36, S200-S208.

6. (a) Bort, G.; Catoen, S.; Borderies, H.; Kebsi, A.; Ballet, S.; Louin, G.; Port, M.; Ferroud, C. Gadolinium-based contrast agents targeted to amyloid aggregates for the early diagnosis of Alzheimer's disease by MRI. Eur. J. Med. Chem. 2014, 87, 843-861; (b) Yong-Sang, J.; Dioury, F.; Meneyrol, V.; Ait-Arsa, I.; Idoumbin, J.-P.; Guibbal, F.; Patché, J.; Gimié, F.; Khantalin, I.; Couprie, J.; Giraud, P.; Ferroud, C.; Jestin, E.; Meilhac, O. Development, synthesis, and ${ }^{68} \mathrm{Ga}$-labeling of a lipophilic complexing agent for atherosclerosis PET imaging. unpublished work to be submitted.

7. Choi, H. S.; Gibbs, S. L.; Lee, J. H.; Kim, S. H.; Ashitate, Y.; Liu, F.; Hyun, H.; Park, G.; Xie, Y.; Bae, S.; Henary, M.; Frangioni, J. V. Targeted zwitterionic near-infrared fluorophores for improved optical imaging. Nat Biotechnol. 2013, 31(2), 148-153.

8. (a) Balajthy, Z. An improved preparation of N-benzyloxycarbonyl-L-lysine methyl ester hydrochloride. Organic Preparations and Procedures International 1991, 23(3), 375-376; (b) Schwenk, S.; Ronco, C.; Oberheide, A.; Arndt, H.-D. Biomimetic synthesis of Urukthapelstatin A by Aza-Wittig ring contraction. Eur. J. Org. Chem., 2016, 28, 4795-4799.

9. Dioury, F.; Ferroud, C.; Guy, A.; Port, M. Synthesis of an hexadentate tricyclic tetraazadiacetic ligand as precursor for MRI contrast enhancement agents. Tetrahedron 2009, 65, 7573-7579.

10. Laurent, S.; Henoumont, C.; Stanicki, D.; Boutry, S.; Lipani, E.; Belaid, S.; Muller, R. N.; Vander Elst, L. Paramagnetic gadolinium complexes. In MRI contrast agents, from molecules to particles; Subramanian T.S., Karthikeyan N., Padmanabhan P., Paulmurugan R., Eds.; Springer: Singapore, 2017; pp. 23-54.

11. Chilla, S. N. M.; Henoumont, C.; Vander Elst, L.; Muller, R. N.; Laurent, S. Importance of DOTA derivatives in bimodal imaging. Isr. J. Chem. 2017, 57, 1-10.

12. Port M. Lipophilic chelates and use thereof in imaging. WO2006100305. 